\title{
Oral Pathology - The Soul of Dentistry
}

\author{
Ishita Singhal ${ }^{1}$, Aparna Dave $^{2}$, Manpreet Arora ${ }^{3}$, Pulin Saluja4, Radhika Rai ${ }^{5}$ \\ 1, 2, 3, 4, 5 Department of Oral Pathology \& Microbiology and Forensic Odontology, \\ S.G.T. Dental College, Hospital \& Research Institute, Gurugram, Haryana, India.
}

\section{ABSTRACT}

\section{BACKGROUND}

Oral and maxillofacial pathology, is the very foundation of dentistry. It is a branch of dentistry where research meets clinical practice with the use of histopathology, cytopathology, haematology, immunohistochemistry, clinical biochemistry, microbiology, molecular science, forensic odontology, and a lot more, making it a unique and rare branch in its own way. It acts as a backbone of dental science, by investigating the causes, processes, and effects of the disease affecting the oral and maxillofacial region. Therefore, this branch requires timely up-gradation to analyse health data, research findings and treatment techniques to offer a diagnostic and therapeutic recommendation for individual patients. The application of digital technology in oral pathology is on the verge of becoming a standard of care, thereby eliminating the need for practising pathology on a conventional microscope. Globally, pathological laboratories need to be more efficient than before. Therefore, automation and digitalization are required to not only increase the efficiency, growth, quality, and capacity but also to standardise the processes. The purpose of this article is to introduce the readers, the incorporation of new ideas and technologies in the lab for a better and faster diagnosis, and would also like to explain the concept of standardizing the method of recording and maintaining the dental or medical records of the patients, and also standardizing the lab reports.

\section{KEY WORDS}

Innovation, Robotics, Digitalization, Artificial Intelligence, Upgradation, Maxillofacial Region
Corresponding Author:

Dr. Aparna Dave,

Prof \& HOD,

Department of Oral Pathology \&

Microbiology and Forensic Odontology,

S. G. T. Dental College, Hospital \& Research Institute, Gurugram, Haryana, India.

E-mail: aparna.dave@sgtuniversity.org

DOI: $10.14260 / j e m d s / 2021 / 666$

How to Cite This Article:

Singhal I, Dave A, Arora M, et al. Oral pathology - the soul of dentistry. J Evolution Med Dent Sci 2021;10(37):3283-3288, DOI: 10.14260/jemds/2021/666

Submission 19-05-2021,

Peer Review 09-08-2021,

Acceptance 16-08-2021,

Published 13-09-2021.

Copyright @ 2021 Ishita Singhal et al. This is an open access article distributed under Creative Commons Attribution License [Attribution 4.0 International (CC BY 4.0)] 


\section{BACKGROUND}

In this $21^{\text {st }}$ century, medicine is becoming challenging day by day with more opportunities. Yet, despite the challenges and opportunities, the recognition of the importance of the oral pathology branch in the minds of the Indian dental fraternity is quite behind. Oral and maxillofacial pathology is a link between basic and clinical science. It understands the problems of a human microscopically and finds out the best possible ways to solve them macroscopically, given the massive area available for researches and exploring the problem regarding the pathophysiology of diseases and preventing its recurrence in future. Therefore, oral pathologists are "The Doctor's Doctor", and act as the epitome of research in dentistry. However, due to the lack of infrastructure and funding for research in dental institutions across the country, the scope of research in this branch is neglected.

The traditional method of pathology that relies solely on the glass slides to render the diagnosis has suspended the patient care, as initial diagnoses and subsequent second opinions often get delayed because it took time for the glass slides or specimens to be delivered physically to the appropriate pathologist. Therefore, to overcome this problem, we require a revolution in diagnosing the problem, digitalising the lab and standardising the records of the patients. It is only by creating innovation in technology, strategies, practices and policies that we will be able to combat local and global healthcare challenges. Incorporation of the latest technologies and advancements in pathology can improve the safety of the patient, service delivery and communications, as well as reduce error rates, lower costs, and better and more efficient use of data. Oral pathology is now at the forefront of the revolution and is entering into an exciting time with new scientific tools and knowledge that are taking pathologists into broader roles of research and correlating diagnoses for the clinicians. So, what does the future hold for pathology in the next generation?

\section{DIGITAL PATHOLOGY}

Digital pathology is growing in the diagnostic realm giving quality assurance, both professionally and technically. ${ }^{1}$ It scans every slide and stores it in the database system, like whole slide imaging (WSI). In the future, sharing of slides between parties on any electronic device can help in studying or managing data or can be used for teleconsultations. This technology helps in primary diagnosis, frozen section diagnosis, and real-time and delayed consultations over a long distance, providing an advantage over conventional glass slides. ${ }^{1}$ Digital pathology also helps the undergraduates, postgraduates and teachers to understand each slide with the help of image analysis software and encourages them for research work.

Often surgeons require opinions for special cases from the pathologists, in such cases telepathology comes in handy. Telepathology is the practice of digital anatomic pathology and clinical pathology at a distance, by transmitting macroscopic and / or microscopic images electronically using telecommunication links for telediagnosis, teleconsultation, distant learning, and research purposes. It enhances the patients' care and efficiency of pathology services between hospitals and academic institutions. ${ }^{2}$

Due to this Covid-19 pandemic, digital pathology is, by now, indisputably the future of the field, as it has hastened the need for remote learning, and we have shifted all of our teachings to whole slide images. Although some are still reluctant to accept its place in the lab due to its cost, legal and regulatory issues, technological drawbacks, resistance from the pathologists, altered infrastructure and above all the lack of universal standards. ${ }^{2}$ However, in many institutions the digital slide image is replacing the conventional method of viewing the slides, as it is more efficient and user friendly, offering the viewers a more comfortable working experience than sitting humped over a microscope. It also allows many people to see the slides on a big screen at once.

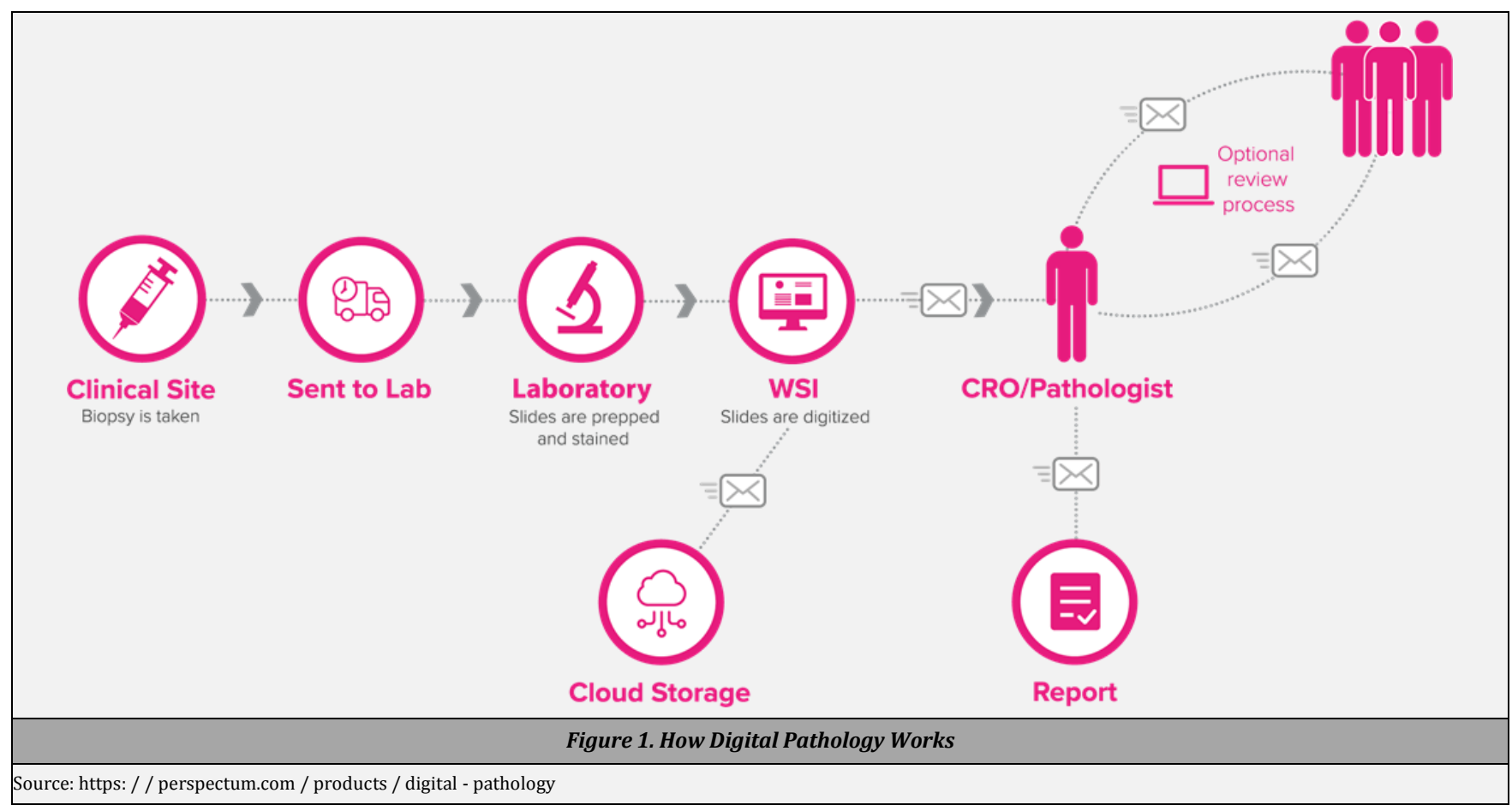




\section{ARTIFICIAL INTELLIGENCE IN PATHOLOGY}

Artificial Intelligence (AI) is the future of pathology that would help in assisting health professionals to cover a variety of human diseases in different organ systems and cope up with the enormous amount of data that digital pathology creates ${ }^{3}$. It must have high quality and legal regulatory criteria at the patient and professional level. However, artificial intelligence does not work alone, and hence requires machine learning (ML) and deep learning (DL). Machine Learning deals with the design, development and evaluation of algorithms that can be learnt from data, to gain knowledge from experience and improve their learning behaviour over time, whereas deep learning is a part of machine learning, which masters compound observable features in images and their associated data. Artificial intelligence, machine learning and deep learning, altogether help solve certain problems, where humans have limited capacities. ${ }^{3}$

The doctors can make a precise clinical diagnosis with the help of machine learning, and the pathologists can spot subtle patterns and provide detailed information to the clinician about the disease. The use of artificial intelligence in pathology helps to analyse digital images and aids in diagnosing the disease, particularly in oncology. Artificial intelligence can also play a handy role like the one augmenting the decision-making capabilities of pathologists, and helping them perform better and faster. ${ }^{3}$ Artificial Intelligence has the potential to improve the efficiency of workflows and the quality of results.

Nevertheless, they cannot replace the pathologist, as it is challenging to translate the technology from academic experiments to clinical use. Any mistake can have serious consequences. After all, the value of human life far outweighs anything and everything. ${ }^{3}$ Therefore, artificial intelligence should have high accuracy performance and should be comprehensible and authentic for the end-users.

\section{ROBOTIC PATHOLOGY}

Until now, we had been examining all the pathological slides in two dimensions through the microscope, but pathologies like cancer have a complex tissue structure, which requires revealing the 3D structures for a better diagnosis. The traditional method of cutting the slides by hand and then placing the tissue between two pieces of glass slides processed only 12 sample slices per hour, which took a lot of time and energy.

Now, a 3D serial section microscopy called Knife-Edge Scanning Microscope (KESM) is available for examining the 3 dimensional nature of the tissue. It captures high-resolution images of thinly sliced layers of tissues and creates digital 3D images of cellular structures. KESM tool uses an automated diamond knife to cut tissue samples at 1000 slices per hour and simultaneously scanning images of each slice to create a 3D tissue model, like that of a CT scan. This technology can automate almost all the work of pathologists, by increasing the speed and efficacy. The high-resolution images provided at multi cubic centimetre scale helps in comparing the 3dimensional nature of vascular networks at whole organ scale and sub-micron resolution. ${ }^{4}$ This technology not only improves the diagnostics but also manages and stores the data, reduces errors, improves quality and helps in teleconsultation also.
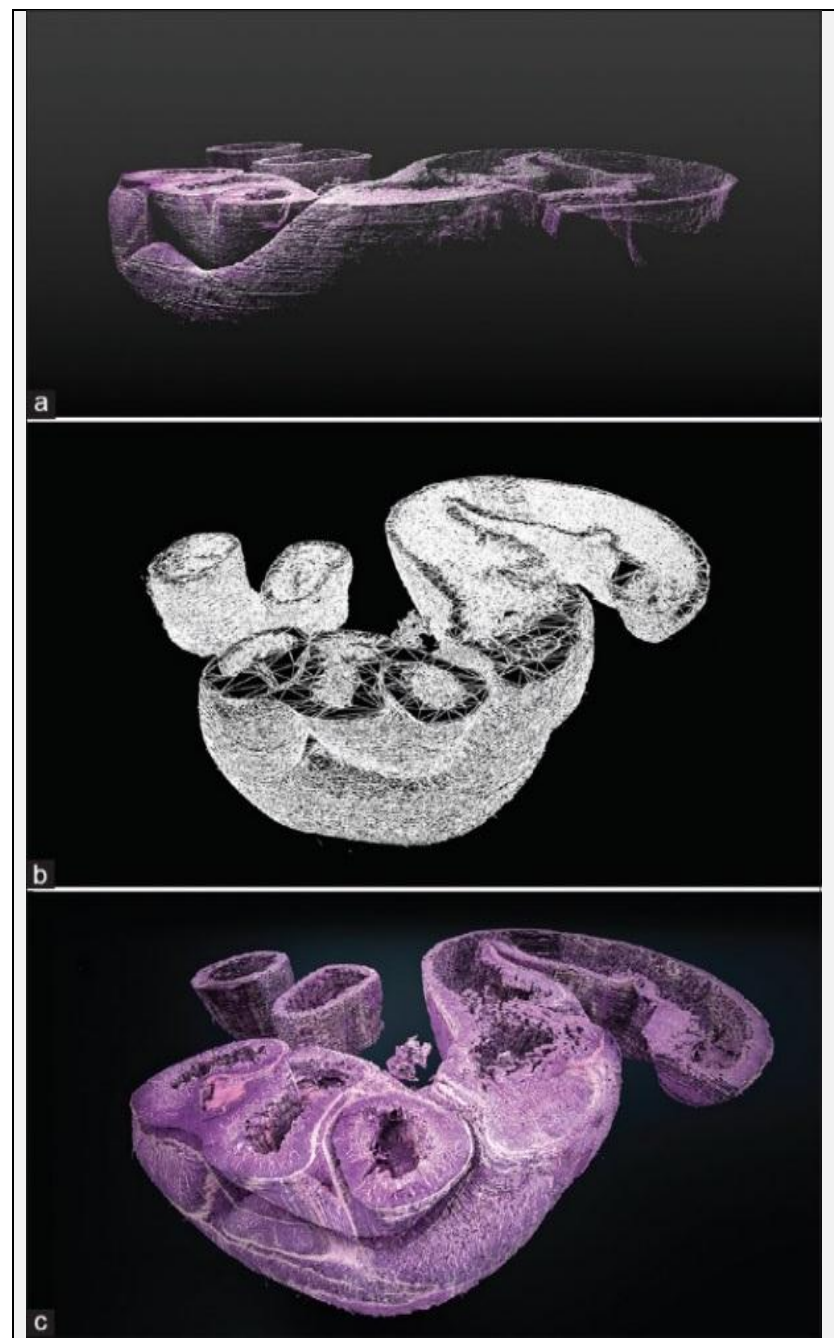

Figure 2. Knife-Edge Scanning Microscope (KESM) is Available for Examining the 3-Dimensional Nature of the Tissue

Source: Farahani N, Braun A, Jutt D, Huffman T, Reder N, Liu Z, Yagi Y, Pantanowitz L. hree-dimensional imaging and scanning: Current and future applications for pathology. J Pathol Inform 2017; 8:36

\section{UPSURGE OF PRECISION MEDICINE}

Administration of the right treatment to the right patient at a right time in the right dose, by increasing the patient's specificity is the major goal of modern medicine. Precision medicine is a changeover from conventional reactive medicine based on symptoms, diagnosis and treatment to a system that targets the disease before it occurs, which means it is only treating diseases after their clinical initiation. It helps in the prevention and treatment of the disease, taking into account the individuals' genes, surrounding environment and lifestyle.

This information will therefore predict accurately that which treatment would be most effective and safe, or possibly how to avert the illness from starting in the first place. Molecular diagnostics, imaging, and analytics are a part of precision medicine. Besides this, it also shifts the emphasis in medicine from reaction to prevention, predicts susceptibility to disease, improves disease detection and progression, customizes disease-prevention strategies, prescribes more effective drugs, avoids prescribing drugs with predictable side 
effects, reduces the time, cost, and failure rate of pharmaceutical clinical trials, and eliminates trial - and - error inefficiencies that inflate health care costs and undermine patient care. ${ }^{5}$

With precision medicine, pathologists will need to play a nimbler role in clinical decision making, by understanding the patient's actual condition and giving a final diagnosis that will be beneficial for the patient. Pathologists will derive the information from various tests like molecular profiling tests or genomic tests and correlate it clinically, and design therapies that will target the specific genes. ${ }^{6}$

For treatment, customized medical products such as drug cocktails produced by pharmacy compounding or customized devices can be involved in precision medicine. The advantage of precision medicine is to prevent harmful drug interactions, reduce costs associated with healthcare, and increase the overall efficiency when prescribing medications. ${ }^{5}$

Precision medicine is not applicable for all, but it is for those who have melanoma, some type of leukaemia, breast cancer, lung cancer, colon cancer, or rectal cancer. Initially, genetic changes in the patient are tested and diagnosed, and then a targeted drug or another treatment for that specific type of cancer is approved. ${ }^{7}$

\section{RAPID ON-SITE EVALUATION [ROSE]}

Rapid on-site evaluation (ROSE) is a clinical service to provide immediate and optimal clinical care for patients having either a superficial or a deep-seated FNA or core biopsy. The cytopathologist and the clinician together perform the procedure, making it an interactive and consultative process. It also provides immediate assurance of adequacy for the minimally invasive FNA biopsy procedure and increases the sensitivity, accuracy and utility of biopsies, thereby improving patient care. ${ }^{8}$

The traditional method of performing a biopsy cannot assure the clinician whether the material obtained is diagnostic or not, but ROSE can provide immediate feedback and communication during the procedure and allow the performing clinician to adapt and modify the biopsy to obtain an adequate diagnostic specimen. ${ }^{8}$

ROSE can help in avoiding multiple biopsies of the same lesion, a repeat biopsy or an inadequate biopsy. Therefore, limiting the use of more invasive diagnostic procedures and delay in diagnosis. It can also reduce the number of direct invasive aspirates, therefore minimizing the use of an overall number of slides. ${ }^{9}$

\section{REGENERATIVE MEDICINE}

Regenerative medicine (RM) is a branch of medicine that develops methods to reproduce and restore organs or tissues, damaged or diseased cells affected by age, disease or trauma in the lab, back to their normal anatomic and physiologic functions by various molecular techniques like 3D bioprinting. ${ }^{10}$ It is a very sensitive procedure, which requires isolation, hygiene, and culturing of cells in a well-equipped laboratory, thereby eliminating the need for organ transplantation. Oral pathologists can easily design disease models using cell lines, animals and organs on a chip, which would help in formulating the exact treatment. This therapy ranges from the regeneration of bone to treatment of malignancies. ${ }^{11}$

The goal of regenerative medicine is to avoid rejection of new tissue by the host immune system, as the immune system also plays a major role in regulating regeneration, both impairing and contributing to the healing process and engraftment. It involves a multidisciplinary approach that applies engineering and life science principles, creating a future for the pathologists of both medical and dental research. ${ }^{10}$

\section{COMPUTER-AIDED DIAGNOSIS (CAD)}

CAD systems support the pathologist in their decision making and reducing the existing observer variation of pathologists. These systems can automatically measure the extent of the cancerous area, grade of cancer, and generate localized cancer maps. CAD systems have evolved with time and are here with new features that focus on biopsies. However, these systems do have some disadvantages as they seem to be less accurate in the grading of cancer and have till now been tested on prostate cancers only. The CAD system can help the pathologists in understanding the growth pattern of a tumour and can focus on specific tumours like the high-grade tumours because in such cases there is loss of differentiation or no structures to detect. ${ }^{12}$

\section{LAB ON A CHIP [LOC]}

LOC is a tiny device used in the laboratory that integrates into a single chip with one or several analyses like biochemical tests, HIV tests, blood tests and DNA tests. It is the splitting of a single drop of blood into various microchannels and initiating thousands of biochemical operations, to get a precise diagnosis. It enables the handling of fluids in low quantities and hence manipulates the biochemical reactions in very small volumes. There is a wide range of applications, which includes human diagnostics, synthesis of chemicals and DNA analysis. This miniaturization is cost-effective, ergonomic, quick in diagnosis, highly accurate and sensitive. It is also equipped with valves, electrodes, pumps, electrical fields and electronics to become a complete diagnostic system. ${ }^{13}$

\section{NANOPATHOLOGY}

Nanopathology is an ultra-specialized branch of pathology, used for the early detection of many diseases of any organ. It explains the etiopathogenesis of diseases associated with inorganic nanoparticles. This branch is useful for studying contagious nanoparticles and to determine the link between unfavourable past exposure and the pathology caused due to it. These nanoparticles can spread through ingestion, inhalation or can also enter through the skin or conjunctiva, and ultimately may enter the blood or lymphatic system, which further distributes to various other organs, having an adverse effect on the respiratory and the cardiovascular system. Scanning electron microscopy with energy disperse spectroscopy (SEM-EDS) can identify these metal-based particles in tissues and body fluids. Recent studies have also 
shown that few nanoparticles can pass through the pores of the nuclear membrane, hence possessing a risk of damage to the cells and genetic information causing mutations in the genes. ${ }^{14}$

Due to the increased demand for screening protocols and the development of certain testing for personalized medicine, there is an increase in histology workload. Automation in the laboratory can be the solution to decrease this workload and save time. It also reduces the requirement for manual intervention. The authentication and verification of all the processes, as well as standardizing the equipment and reagents used, are the first steps to automation. Automation and digitalization can start right from receiving the specimen until reporting. However, the problems that occur are the finance and rapid sample analysis. New technology has to be invented for rapid analysis, where the report can be available within 24 hours of a biopsy. The goal of automation is to decrease the workload, save time and energy. It has to be user friendly and efficient also, so that, it is beneficial for both the staff and the patients.

Pathologists work starts, as soon as the tissue is excised from the patient's body. Automation and tracking can start when receiving the sample. The specimen receiving time and temperature at which the specimen was stored and transported will be recorded for standardisation. A barcode can be assigned to every form, block and slide to track it at every step, right up to the time the sample is concluded. All the data can then be integrated into the Laboratory Information Management System (LIMS). This information can provide the easy workflow, identification of errors and quality indication information.

All the laboratories need to standardize their tests to interpret the results accurately and adopt clinical protocols. Every lab uses a different reference unit and a different range for every test, which can be very confusing for the lab technicians and the health care professionals, and therefore result in misinterpreted lab results, causing the patient to get their tests repeated. Therefore, standardization would benefit the laboratories through improved quality and regulation and reduced prolixity and errors. Using the same methodology and instrumentation to perform the tests, would make the same testing policies, procedures, and best practices all over the world.

Electronic health record streamlines the laboratory information electronically for every test, providing a code for the test, name of the procedure, reference interval, critical values, results, and location. Patients will be beneficial, as they would not require to carry all the reports every time and everywhere, and the reports will also not get misplaced and they can also consult any doctor worldwide by sharing their data electronically at any time; Doctors can view the past and present case history of the patient easily, and accordingly plan the treatment.

As the health care system continues to develop, standardization of lab will remain a work in progress through phases of developing and sharing best practices and learning and working together as one unified laboratory. System laboratory directors with specialization in different laboratory areas can provide a way to re-engineer the processes and ensure that all local management teams align with the systemized approach.

In India, all the health records of a patient like lab reports (X - rays, MRI, biopsy, biochemical analysis, culture tests, etc.) and prescriptions can be linked with the Aadhaar card, and the patient will have an option to share his data with the hospitals or private clinics. A one-time password on his / her registered mobile can be generated each time the patient wants to share the data. This would provide privacy and uniformity. By this method, the doctor can view and discuss with his / her colleagues about the patient's treatment and prognosis easily. This system will minimize errors in reports, standardize all the labs, maintain records at one place, register all doctors and patients at one place, and would also provide an exact health status of the country. 10

\section{CONCLUSIONS}

It is important that the dentist and other health professionals get to know more about the uniqueness of this field since oral pathologists not only are capable of diagnosing various diseases and oral cancers, their unique training and experiences are capable of providing specialized services in the field of dentistry.

Financial or other competing interests: None.

Disclosure forms provided by the authors are available with the full text of this article at jemds.com.

\section{REFERENCES}

[1] Luo W, Hassell LA. Use cases for digital pathology. In: Digital Pathology. Cham: Springer 2016:5-15.

[2] Farahani N, Pantanowitz L. Overview of telepathology. Surg Pathol Clin 2015;8(2):223-31.

[3] Holzinger A, Goebel R, Mengel $M$, et al. Artificial intelligence and machine learning for digital pathology: state-of-the-art and future challenges. Springer Nature 2020.

[4] Pesavento MJ, Miller C, Pelton K, et al. Knife-edge scanning microscopy for bright-field multi-cubic centimetre analysis of the microvasculature. Microscopy Today 2017;25(4):14-21.

[5] Ginsburg GS, Phillips KA. Precision medicine: from science to value. Health Aff (Millwood) 2018;37(5):694701.

[6] Mccall SJ, Dry SM. Precision pathology as part of precision medicine: are we optimizing patients interests in prioritizing use of limited tissue samples? JCO Precision Oncology 2019;3:1-6.

[7] Gameiro GR, Sinkunas V, Liguori GR, et al. Precision medicine: changing the way we think about healthcare. Clinics (Sao Paulo) 2018;73:e723.

[8] Sauter JL, Chen Y, Alex D, et al. Results from the 2019 American Society of Cytopathology survey on rapid onsite evaluation (ROSE)-part 2: subjective views among the cytopathology community. J Am Soc Cytopathol 2020;9(6):570-8.

[9] Anila KR, Nayak N, Venugopal M, et al. Role of rapid onsite evaluation in CT-guided fine needle aspiration cytology of lung nodules. J Cytol 2018;35(4):229-32.

[10] Mao AS, Mooney DJ. Regenerative medicine: current therapies and future directions. Proc Natl Acad Sci 2015;112(47):14452-9. 
[11] Sunil PM. Regenerative medicine-a new horizon for an oral pathologist. J Oral Maxillofac Pathol 2020;24(1):112.

[12] Jansen I, Lucas M, Savci-Heijink CD, et al. Histopathology: ditch the slides, because digital and 3D are on show. World J Urol 2018;36(4):549-55.
[13] Yılmaz B, Yılmaz F. Lab-on-a-chip technology and its applications. In: Omics Technologies and Bioengineering. Academic Press 2018:145-53.

[14] Dvořáčková J, Bielniková H, Mačák J. Nanopathology as a new scientific discipline. Minireview. Cesk Patol 2013;49(1):46-50. 